\title{
1 MHC genotyping from rhesus macaque exome sequences
}

3 John R. Caskey ${ }^{1}$, Roger W. Wiseman ${ }^{1,2}$, Julie A. Karl ${ }^{2}$, David A. Baker ${ }^{1}$, Taylor Lee ${ }^{1}$, Muthuswamy

4 Raveendran $^{3}$, R. Alan Harris ${ }^{3}$, Jianhong $\mathrm{Hu}^{3}$, Donna M. Muzny ${ }^{3}$,Jeffrey Rogers ${ }^{3}$, David H. O’Connor ${ }^{1,2}$

$5 \quad{ }^{1}$ Wisconsin National Primate Research Center, University of Wisconsin-Madison, Madison, WI 53715,

6 USA

$7 \quad{ }^{2}$ Department of Pathology and Laboratory Medicine, University of Wisconsin-Madison, Madison, WI

8 53705, USA

$9{ }^{3}$ Human Genome Sequencing Center, Baylor College of Medicine, Houston, TX 77030, USA

10 Corresponding author: Dr. David H. O’Connor (doconnor@primate.wisc.edu; +1 608890 0845)

\begin{tabular}{|l|l|}
\hline Author & ORCID \\
\hline Caskey & $0000-0001-5665-524 \mathrm{X}$ \\
\hline Wiseman & $0000-0002-7682-7085$ \\
\hline Karl & $0000-0002-4447-4721$ \\
\hline Baker & $0000-0002-6811-8238$ \\
\hline Lee & $0000-0002-8768-4358$ \\
\hline Raveendran & $0000-0001-6185-4059$ \\
\hline Jianghong & $0000-0002-6318-8595$ \\
\hline Harris & $0000-0002-7333-4752$ \\
\hline Rogers & $0000-0002-7374-6490$ \\
\hline O'Connor & $0000-0003-2139-470 \mathrm{X}$ \\
\hline
\end{tabular}

11

12 Keywords: Macaca mulatta, exome, major histocompatibility complex 


\section{Abstract}

14 Indian rhesus macaque major histocompatibility complex (MHC) variation can influence the outcomes of

15 transplantation and infectious disease studies. Frequently, rhesus macaques are MHC genotyped to identify variants

16 that could account for unexpected results. Since the MHC is only one region in the genome where variation could

17 impact experimental outcomes, strategies for simultaneously profiling variation in the macaque MHC and the

18 remainder of the protein coding genome would be useful. Here we introduce macaque exome sequence (MES)

19 genotyping, in which MHC class I and class II genotypes are determined with high confidence using target-

20 enrichment probes that are enriched for MHC sequences. For a cohort of 27 Indian rhesus macaques, we describe

21 two methods for obtaining MHC genotypes from MES data and demonstrate that the MHC class I and class II

22 genotyping results obtained with these methods are $98.1 \%$ and $98.7 \%$ concordant, respectively, with expected MHC

23 genotypes. In contrast, conventional MHC genotyping results obtained by deep sequencing of short multiplex PCR

24 amplicons were only $92.6 \%$ concordant with expectations for this cohort. 


\section{Introduction}

31 The major histocompatibility complex (MHC) is an intensively studied set of genes in macaques (Shiina et al. 2017;

32 Wiseman et al. 2013). The genomic MHC region contains clusters of genes that encode the MHC class I complex and the MHC class II complex. Cells use MHC class I molecules to present intracellular peptides to immune cells like the CD8+ T cell or natural killer cells (Garcia and Adams 2005). MHC class I molecules accommodate intracellular peptides of varying specificity by having diverse amino acid sequences in the $\alpha 1$ and $\alpha 2$ subunits, which form the peptide-binding cleft (Silver and Watkins 2017; Loffredo et al. 2009). These $\alpha 1$ and $\alpha 2$ subunits correspond to exons 2 and 3, respectively, of an MHC class I gene (Malissen et al. 1982). Most of the polymorphisms that distinguish MHC class I alleles are concentrated in exons 2 and 3 (Williams 2001). Thousands of individual MHC allelic variants have been identified in the three most widely used macaque species for biomedical research: rhesus (Macaca mulatta), cynomolgus (Macaca fascicularis), and pig-tailed macaques (Macaca nemestrina) (Semler et al. 2018; Karl et al. 2017; Maccari et al. 2017). the HLA-A, HLA-B, and HLA-C genes on each chromosome (Daza-Vamenta et al. 2004; Shiina et al. 2017). In contrast, macaques have a variable number of genes on each chromosome that encode MHC class I MHC-A and MHC-B proteins, and macaques lack an HLA-C orthologue (Daza-Vamenta et al. 2004; Shiina et al. 2017; Wiseman et al. 2013). Initial approaches to genotype macaque MHC class I relied on using sequence-specific PCR oligonucleotides to test for the presence or absence of individual alleles (Kaizu et al. 2007). More recently, deep sequencing genomic DNA or complementary DNA PCR amplicons spanning a highly variable region of exon 2 has become commonplace (Wiseman et al. 2009; Karl et al. 2013). Amplicon sequences can be used to genotype groups

50 of closely related MHC class I alleles, which are denoted as lineages. For example, an amplicon deep sequence that

51 corresponds to the rhesus macaque $М а т и-A 1 * 001$ lineage demonstrates that an animal possesses $M a m u-A 1 * 001: 01$,

52 Мати-A1*001:02, or another closely related variant that has not yet been identified. This lineage-level reporting of

53 MHC class I genotypes can be sufficient for designing experiments where certain MHC class I genotypes need to be 54 matched between animals, balanced among experimental groups, or excluded entirely from a study (Loffredo et al. 
2007; Loffredo et al. 2009; Muhl et al. 2002; Karl et al. 2013; Wiseman et al. 2009; Nomura et al. 2012; Mothe et al. 2003).

Amplicon deep sequencing can also be used for MHC class II genotyping. The human HLA class II genes DQA1, $D Q B 1, D P A 1$, and $D P B 1$ have direct orthologues in macaques (Otting et al. 2017). Both macaques and humans have a variable number of MHC class II DRB genes on a single chromosome, while the DRA gene is oligomorphic (Daza-Vamenta et al. 2004; Shiina et al. 2017), and typically is not used for genotyping purposes. MHC class II molecules are members of the immunoglobulin superfamily, but they differ from MHC class I in several ways. The MHC class II complex is comprised of an $\alpha$ and $\beta$ subunit heterodimer, and separate genes encode each MHC class II $\alpha$ and $\beta$ subunit (Brown et al. 1993). Highly polymorphic regions that are diagnostic for MHC class II allele lineages can be PCR amplified in a manner that is similar to the MHC class I genotyping. The most extensive polymorphism among alleles of the MHC class II genes is found in exon 2 (Williams 2001). Each of the DRB, $D Q A 1, D Q B 1, D P A 1$ and $D P B 1$ polymorphic MHC class II genes are sufficiently divergent that separate PCR amplicons are required for deep sequencing (Karl et al. 2014).

Comprehensive MHC class I and class II genotyping of macaques by amplicon deep sequencing requires preparation of six separate PCR amplicons for MHC class I and class II DRB, DQA1, DQB1, DPA1, and DPB1 (Karl et al. 2014; Karl et al. 2017). Despite this complexity, a major advantage to amplicon deep sequencing has been its cost effectiveness. The output from a single MiSeq sequencing run can be used to determine the MHC class I and MHC class II genotypes for up to 192 macaque samples. In recent years, improved sequencing hardware and software have prompted new approaches to MHC genotyping. Instead of a utilizing PCR amplicons of variable gene regions to genotype samples, researchers can use human whole exome sequencing (WES) and whole genome sequencing (WGS) datasets to determine HLA genotypes (Xie et al. 2017; Kishikawa et al. 2019; Yang et a.1 2014; Posey et al. 2016). Likewise, target capture approaches have been described for HLA genotyping with next-generation sequencing (Cao et al. 2013; Wittig et al. 2015). In contrast, MHC genotyping of macaques from WGS (Xue et al. 2016; Bimber et al. 2017; de Manuel et al. 2018) or whole exome sequence (Vallender 2011; Cornish et al. 2016) datasets have not been reported to date. This reflects the challenges presented by mapping short sequence reads against complex, duplicated gene families like the MHC class I genes of macaques where genomic reference sequences and reference databases of known alleles are incomplete (Zimin et al. 2014; Maccari et al. 2017). In an 
82 era where the per-base cost of sequencing is dropping rapidly, we explored the feasibility of obtaining whole exome

83 sequencing data while maintaining parity of results with the traditional MHC PCR amplicon approach.

84 Here we introduce MHC genotyping via macaque exome sequencing (MES), which is an exome sequencing-based

85 workflow for comprehensive MHC class I and class II genotyping in macaques. This workflow uses a commercially

86 available human exome target-capture enrichment kit in conjunction with specialized spike-in target-capture probes

87 to specifically cope with the high copy number of macaque MHC genes. We show that accuracy of Indian rhesus

88 macaque MHC class I and class II results from this workflow are comparable to conventional MiSeq genotyping

89 when using exon 2 reference sequences. 


\section{$91 \quad$ Methods}

92

93

94

95

96

97

98

99

100

101

102

103

104

105

106

107

108

109

110

111

112

113

114

\section{Animals}

Twenty-seven whole blood samples were collected from Indian rhesus macaques (Macaca mulatta). Five of these samples came from a breeding group of animals living at the Wisconsin National Primate Research Center (WNPRC). The remaining 22 samples were provided by Dr. Michele Di Mascio from the National Institutes of Health's National Institutes of Allergy and Infectious Diseases. Blood sampling was performed under anesthesia and in accordance with the regulations and guidelines outlined in the Animal Welfare Act, the Guide for the Care and Use of Laboratory Animals, and the Weatherall report (Animal Welfare Act 1966; Weatherall 2006).

\section{Data}

Exome sequence datasets have been deposited in the sequence read archive (SRA) under BioProjects PRJNA529708 and PRJNA527214. Fasta reference sequences used for MHC genotyping, and sequence analysis scripts are available from https://go.wisc.edu/jb0926.

\section{MHC class I and class II genotyping by amplicon deep sequencing}

\section{Genomic DNA was isolated from $250 \mu \mathrm{L}$ of whole blood using a Maxwell@ 48 LEV Blood DNA Kit (Promega}

Corporation, Fitchburg, WI). Following isolation, DNA concentrations were determined with a Nanodrop 2000 and samples were normalized to $60 \mathrm{ng} / \mathrm{ul}$. MHC class I and class II PCR amplicons were generated using exon 2-specific primers with adapters (CS1 and CS2) necessary for 4-primer amplicon tagging with the Fluidigm Access Array ${ }^{\mathrm{TM}}$ System (Fluidigm, San Francisco, CA, USA) by previously described methods (Karl et al. 2017; Karl et al. 2014). Pooled PCR products were purified using the AMPure XP beads (Agencourt Bioscience Corporation, Beverly, MA, USA) and quantified using the Quant-iT dsDNA HS Assay kit with a Qubit fluorometer (Invitrogen, Carlsbad, CA, USA), following the manufacturer's protocols. The MHC exon 2 genotyping amplicon pools were sequenced on an Illumina MiSeq instrument (San Diego, CA, USA) as previously described (Karl et al. 2017).

Analysis of the MiSeq exon 2 genotyping amplicon sequences was performed using a custom Python workflow. The workflow contained a step to remove oligonucleotide primers and sequencing adapters with bbduk, merge a step to 
115

116

117

118

119

120

121

122

123

124

125

126

127

128

129

130

131

132

133

134

135

136

137

138

139

140

141

reads bbmerge, a step to identify unique sequences/remove chimeras with USEARCH, and finally mapping unique reads against a deduplicated reference database of rhesus macaque partial MHC class I and class II exon 2 sequences with bbmap (Bushnell et al. 2017; Edgar 2010). The database was derived from sequences in the IPD-MHC NHP database downloaded from the European Bioinformatics Institute website (Maccari et al. 2017). For this publication, we define "IPD exon 2" as this database of reference sequences. SAM output files from bbmap were parsed with the Python package pandas to enumerate the reads from each animal that were identical to IPD exon 2 reference sequences. Mamu-A, $-B,-D R B,-D Q A 1,-D Q B 1,-D P A 1$ and $-D P B 1$ lineage-level haplotypes were inferred for each of the samples with a semi-automated custom workflow that identifies diagnostic alleles associated with previously defined rhesus macaque haplotypes (Karl et al. 2013; Otting et al. 2017).

\section{MHC class I and class II genotyping by exome sequencing}

Genomic DNA was isolated as described above and shipped to the Human Genome Sequencing Center at the Baylor College of Medicine. MHC and exon-containing genomic DNA fragments were selectively enriched using a custom target-capture probeset. Genome-wide exons were captured using SeqCap EZ HGSC VCRome2.1, an optimized human clinical exome probeset (Clark et al. 2013, Yang et al. 2013). SeqCap EZ HGSC VCRome2.1 contains probes designed to enrich 23,585 human genes and 189,028 non-overlapping exons. A low coverage audit was performed to identify rhesus macaque exons inferred from the reference genome rheMac2 that were not sufficiently enriched $(<20 x$ coverage) with these human probes, and an additional 22,884 rhesus macaque exons were incorporated into the genotyping probe design (Prall et al. 2017). Finally, and most importantly for MHC analyses, we modified the SeqCap EZ Design: Human MHC Design to selectively enrich MHC class I and class II sequences. This previous design was prepared by the Beijing Genome Institute in collaboration with Roche/Nimblegen and it targeted the complete 4.97Mb HLA region with non-redundant probes designed against 8 fully sequenced HLA haplotypes (Horton et al. 2008; Cao et al. 2013). For our macaque studies, we prepared a minimal MHC target capture design using a subset of these probes that are based on all functional HLA class I (HLA-A, $-B,-C$, and $-E)$ and class II (HLA-DRA, -DRB1, -DRB3, -DRB4, -DRB5, -DQA1, -DQB1, -DPA1 and -DPB1) genes. Probes were included to capture complete gene sequences (exons + introns +3 ' UTR) as well as $\sim 1 \mathrm{~kb}$ of 5 ' upstream flanking sequence. The BED file of rhesus rheMac2 target coordinates lifted over to rheMac8 was used to prepare this combined minimal MHC and supplemental rhesus spike-in probe design. Because derivation of MHC results from 
142

143

144

145

146

147

148

149

150

151

152

153

154

155

156

157

158

159

160

161

162

163

164

165

166

167

genotyping is paramount, we used a ratio of 2.5x spike-in probes to 1x VCRom2.1 probes. The supplemental probes for MHC and rhesus macaque were a single reagent, and the MHC-specific probes only constituted $609 \mathrm{~kb}$ of the 37.9 Mb probes (Prall et al. 2017).

An Illumina paired-end pre-capture library was constructed with 750 nanograms of DNA, as described by the Baylor College of Medicine Human Genome Sequencing Center. Pre-capture libraries were pooled into 10-plex library pools for target capture according to the manufacturer's protocol. Samples were pooled in 10-plex sequence capture library pools for 151 bp paired-end sequencing in a single lane of an S4 flow cell on an Illumina Novaseq 6000 at the Baylor College of Medicine Human Genome Sequencing Center.

\section{Enumeration of MHC reads in exome data}

The effective enrichment of MHC reads in the exome datasets was calculated by mapping the reads of each sample's exome dataset against an individual genomic reference file for each individual locus: $H L A-A$ exons 2-3 (NCBI Gene ID: 3105) and HLA-E exons 2-3 (Gene ID: 3133); HLA-DPAl exons 2-4 (Gene ID: 3113); HLA-DPB1 exons 2-4 (Gene ID: 3115); HLA-DQA1 exons 2-4 (Gene ID: 3117); HLA-DQA2 exons 2-4 (Gene ID: 3118); HLA-DQB1 exons 2-4 (Gene ID: 3119); HLA-DQB2 exons 2-4 (Gene ID: 3120); HLA-DRB1 exons 2-4 (Gene ID: 3123), HLADRB3 exons 2-4 (Gene ID: 3125), HLA-DRB4 exons 2-4 (Gene ID: 3126) and HLA-DRB5 exons 2-4 (Gene ID: 3127). This mapping was done by using bbmap with default parameters, which corresponds to a minimum alignment identity of approximately 76\% (Bushnell et al. 2017). Empirically, these mapping parameters are sufficient to map macaque MHC reads to their human orthologues. Mapped reads were written to a new fastq file using bbmap's outm= parameter. To quantify the total number of reads in a sample and the number of reads extracted with our reference file, we created a custom Python script, which is available to download.

\section{MHC genotyping from exome data}

Two complementary data analysis strategies were employed to analyze the exome sequence data, and to verify reproducibility and confidence in the MHC genotyping results. For accuracy and quantification purposes, the expected MHC genotypes for each animal were established based on concordance among at least two out of the three described strategies and biological plausibility, e.g., no more than two alleles per Mamu-DQA1, -DQB1, $D P A 1$, or- $D P B 1$ locus. 
The Diagnostic Sub-Region (DSR) was an intra-allelic region that encompassed polymorphisms, and these polymorphisms were distinguishable from alleles with similar sequences. Therefore, this method ensured the DSR

171 was captured in at least one read for each called allele. MHC class I and class II reads initially were extracted from

172 each animal by mapping the FASTQ reads to HLA class I and class II reference sequences containing exons 2-3 and

173 exons 2-4, respectively, plus the intervening intron(s) as described above in 'Enumeration of MHC reads in exome

174 data'. Reads were mapped to these reference sequences using bbmap with default parameters and the parameter

175 (qtrim=lr) (Bushnell et al. 2017).

Following extraction of MHC reads from the total exome sequences, the MHC reads were prepared for assembly using a modified version of a data pre-processing pipeline, which included tools from the BBTools package (Bushnell et al. 2017). Briefly, optical duplicates and reads from low-quality regions of the sequencing run were removed. Next, Illumina sequencing adapters were trimmed from the ends of sequencing reads. Any residual spikein or PhiX sequences that inadvertently survived mapping to HLA class I and class II were then removed. Three rounds of error-correction and read merging were performed to create high-confidence merged reads that were wellsupported by common kmers found in the extracted MHC reads. The error-corrected reads were not merged with a minimum overlap, but instead were separately mapped against the IPD exon 2 sequences using bbmapskimmer. The default settings for the software tool bbmapskimmer were used with the following modified parameters (semiperfectmode $=\mathrm{t}$ ambiguous $=$ all ssa=t maxsites $=50000$ maxsites $2=50000$ expectedsites $=50000)($ Bushnell et al . 2017). The semiperfectmode setting accepted reads with perfect matches, as well as reads that extended off the end of contigs for no more than half of the length of the mapped read segment. The ambiguous setting and the 'expectedsites' setting reported the first 50,000 matched read segments that met the 'semiperfectmode' filtering, which was set exceedingly high in order to exhaustively map our IPD exon 2 reference file of $\sim 1600$ alleles. Using

190 semiperfect mode, these were the segment sequences with the longest matching length. This output file included all

191 mapped reads to all IPD exon 2 reference allele sequences. 
192 We then used samtools mpileup with the settings (-A -a --ff UNMAP -X -B -q 0 -Q 0) on the bbmapskimmer output

193 to calculate a depth of coverage at each position for each IPD exon 2 sequence. Next, we removed any aligned reads

194 to the IPD exon 2 database sequences that contained less than a minimum depth of coverage of two across the entire

195 reference sequence. For ambiguously-mapped reads, the alignments with the longest-matching region were selected

196 for further analysis; ties among alignments were counted multiple times. To reduce the number of false positives, we

197 used Python pandas to only report database sequence matches that had at least one unambiguously mapped read.

198 Based on these mapping parameters, unambiguously-mapped reads must span the DSR. The MHC genotypes from

199 this method were reported as the minimum depth of coverage for each IPD exon 2 database sequence per animal.

Strategy 2: De novo reconstruction of $M H C$ sequences from exome reads

201 Most de novo sequence assemblers have been optimized for resolving long contigs, and are tolerant of small 202 sequence mismatches that can otherwise fragment assemblies. In the case of MHC alleles, however, such closely 203 related sequences were often biologically distinct. Because of the gene duplications in the macaque MHC, there 204 were many valid MHC contigs that could be assembled from a single sample within the exome data. This is conceptually similar to the computational challenge of assembling viral haplotypes, where rapidly evolving viruses

206 such as human immunodeficiency virus accumulate variants that frequently co-segregate as minor populations 207 within an infected person (Baaijens et al. 2017). Therefore, we utilized the overlap assembly algorithm SAVAGE, 208 originally designed to reconstruct viral haplotypes, to reconstruct MHC allele sequences from exome reads (Baaijens 209 et al. 2017). Similar to Strategy 1 above, HLA-mapped reads were pre-processed using the BBTools package to 210 remove low quality reads and optical duplicates. Next, adapters were trimmed, residual spike-in and PhiX sequences 211 were removed, three steps of error-correction took place, and reads were merged. These merged reads, as well as 212 high-confidence unmerged paired-end reads that could not be grouped into an overlapping merged read, were used 213 for SAVAGE assembly. The following workflow was implemented in a reproducible snakemake workflow that fully 214 documents parameter selection (Koster and Rahman 2012), and is available upon request.

215 SAVAGE is designed to construct individual haplotypes from the overlap graph of individual reads. We processed 216 the totality of sequence data in a single patch in order to maximize the sensitivity of the contiguous reconstruction 217 with parameter '--split 1', as well as the parameter '--revcomp' to handle reverse complement reads. The IPD exon 2 
218 reference database that was used for MiSeq and DSR genotyping was also used to assess the quality of SAVAGE

219 genotypes. The IPD sequences were mapped to contigs produced by SAVAGE using sensitive parameters in bbmap

220 (minlength $=100$ vslow $=t$ subfilter $=0$ indelfilter $=0$ lengthtag=t ignorefrequentkmers $=$ t kfilter $=100)$ designed to

221 identify sequences that perfectly match SAVAGE contigs. A post-processing script refined these mappings, and

222 only retained the mappings where the length of the mapped region was the same as the length of the IPD exon 2

223 sequence. These mappings indicated where the reference database sequence was fully and exactly contained within a

224 SAVAGE contig. 


\section{Results}

\section{MHC reads are efficiently enriched using target-capture probes}

230 We designed a custom target enrichment probeset that accounts for the extensive duplication of macaque MHC

231 genes (Prall et al. 2017). A tripartite target capture system was used in this study. The first component is SeqCap EZ

232 HGSC VCRome2.1, an optimized human clinical exome probeset. Since macaques are closely related to humans,

233 SeqCap EZ HGSC VCRome2.1 can also be used in macaques, though some sequences that are most divergent

234 between macaques and humans are not efficiently captured. The second component of the target capture system is

235 an additional 22,884 rhesus macaque exon sequences that were not effectively captured with the SeqCap EZ HGSC

236 VCRome2.1 reagent. The third component is a collection of probes designed to specifically enrich macaque MHC

237 class I and class II sequences. These probes span the full length of HLA class I and class II genes including introns,

238 3' UTRs, and approximately 1,000 bp of flanking 5' sequence.

239 We obtained a median coverage of $100 \mathrm{X}$ for the target exon sequences across the genome with $>20 \mathrm{X}$ coverage for

$24094.97 \%$ of bases that were targeted in this study. As illustrated in Table 1, an average of 70,549,789 Illumina

241 sequence reads per sample were obtained for the 27 animals evaluated in this study. These reads were mapped

242 against reference files containing representative genomic HLA exons 2 -3 for HLA-A, and HLA-E exons 2 - 4 for

$243 \quad H L A-D R A,-D R B 1,-D R B 3,-D R B 4,-D R B 5,-D Q A 1,-D Q B 1,-D P A 1$ and -DPB1 sequences. We identified an

244 average of 269,057 MHC class I and class II sequence reads per sample which corresponds to an average of $0.37 \%$

245 of the total sequence reads evaluated per sample (Table 1). In a previous study by Ericsen et al. (Ericsen et al.

246 2014), we found that MHC sequences only accounted for an average of $0.13 \%$ of the total Illumina sequence reads

247 that were evaluated per animal when the standard HGSC VCRome2.1 panel was used alone for target capture

248 (Supplementary Table 1). Thus, we achieved an almost three-fold increase of MHC genomic sequences after

249 inclusion of the spike-in probes for target capture compared to use of the VCRome2.1 probeset alone.

250 MHC genotypes defined from target-enriched genomic DNA are comparable to those obtained by

251 amplicon deep sequencing 
We hypothesized that MHC genotypes derived from target-enriched genomic sequence would be comparable in accuracy to MHC genotypes derived from conventional amplicon deep sequencing. MHC class I and class II PCR amplicons were generated from the same 27 animals and deep sequenced on an Illumina MiSeq. Representative MHC class I and class II genotypes supported by the amplicon data are shown in Figure 1 and Figure 2 , respectively. These figures illustrate genotypes from a pedigreed family of Indian rhesus macaques: a Sire, a Dam, and three Progeny that are paternal half-siblings. Comprehensive genotypes for all 27 Indian rhesus macaques evaluated in this study are shown in Supplementary Figure 1.

The superset of alleles supported by at least two of the methods described in this manuscript (MiSeq amplicon genotyping, DSR genotyping of individual exome sequencing reads, or SAVAGE genotyping of contigs derived

261 from exome data), was considered to be the expected MHC genotypes for these animals. A complication for this 262 assertion was certain genotypes were difficult for MiSeq analysis to report correctly. As we have shown previously 263 (Karl et al. 2017), the number of reads supporting each genotyping call was highly variable, ranging from tens of 264 reads to thousands of reads per allele. A small subset of allelic variants contained nucleotide substitutions in their 265 sequences within the binding sites of the PCR oligonucleotides that interfere with efficient PCR amplification. This 266 result is exemplified when members of the Mamu-B11L*01 allele lineage were closely examined. Members of the 267 Мати-B11L*01 allele lineage have two nucleotide substitutions relative to the 5' oligonucleotide that was used to 268 generate MHC class I amplicons, so these Mamu-B11L*01 sequences were routinely absent in MiSeq genotypes

269 (Figure 1). Likewise, the Mamu-DPA1*11:01 allele has four nucleotide substitutions relative to the oligonucleotide 270 pair used to generate DPA1 amplicons for MiSeq genotyping (Figure 2).

These nucleotide substitutions do not fully account for all differences in the abundance of sequence reads for each allele. False negatives were also noted for certain allele lineages, such as $M a m u-B^{*} 074$ and $M a m u-B * 098$, which exhibited significantly diminished PCR efficiency despite being completely matched with the oligonucleotides used for amplification (Figure 1). False positive genotyping calls were also noted in the MiSeq assay that resulted from intermolecular recombination during the PCR process. This was exemplified by read support for the presence of 
$B^{*} 007 g 1$ allele group that was determined to be present in this pair of animals by a single nucleotide variant at the extreme 5' end of the class I genotyping amplicon. Chimeric PCR products equivalent to the Mamu-B*007:07 sequence were formed between the 3 ' portion of the $M a m u-B * 007 g 1$ sequence and other allelic variants in these animals with this 5' SNP. Taken together, these results illustrate that the MiSeq amplicon genotyping, while generally reflective of an animal's MHC genotype, can yield both false positive and false negative results. When compared to the expected genotypes for this dataset, MiSeq amplicon genotyping has $90.4 \%$ MHC class I and 97.8\% MHC class II concordance (Table 2).

Two separate strategies were used to derive MHC genotypes from target-capture data. The DSR analysis was a straightforward extension of the methodology used for deriving genotypes from MiSeq amplicons. MHC sequence reads were extracted from the total exome dataset. A simplified workflow that mapped those MHC reads to

287 reference sequences, and then assigned the genotypes based on the presence of exome reads overlapping each 288 position of the reference sequence, was problematic. This simplified workflow was extremely vulnerable to false 289 positive genotypes, and the similarities among different MHC sequences often enabled those reads to map to 290 multiple different alleles (Wiseman et al. 2013). Two or more reads each could partially match a portion of a 291 sequence in the IPD exon 2 database, which could complement each other to provide support for an allele that was 292 not biologically relevant. As discussed in the Methods, the DSR encompassed polymorphisms that discriminated 293 among closely related alleles by requiring at least one mapped read to unambiguously map to the corresponding 294 allele within the IPD exon 2 sequences. This strategy can identify specific polymorphisms of interest among the IPD 295 exon 2 sequences to produce genotypes for MHC class I and MHC class II (Figures 1, 2 and Supplementary

296 Figure 1). For the 27 animals evaluated in this study, DSR was $97.3 \%$ and $100 \%$ concordant with expected MHC 297 class I and class II genotypes, respectively (Table 2).

The DSR strategy did not consider sequences that were not among the IPD exon 2 sequences and this lack of consideration contributes to overcalled alleles. The apparent Mamu-A1*059:01 allele in Dam r05029 was erroneously derived from reads that were from $M a m u-A 1 * 004 g 1$ and an unknown allele that was not among the 

iterations of the IPD database.

305 The second approach for determining genotypes performed de novo assembly on MHC class I and class II reads

306 from each sample. The resulting assembled contigs were then mapped against IPD exon 2 reference sequences to

307 define perfectly matching contigs. Because most assemblers were not tuned for the challenge of assembling large

308 numbers of contigs that differ from one another by as little as $1 \mathrm{bp}$, we relied on an assembler, SAVAGE, originally

309 designed to reconstruct viral sequencing haplotypes. As shown in Figures 1, 2 and Supplementary Figure 1,

310 contigs produced by SAVAGE matched the expected genotypes. Unlike the DSR method, SAVAGE assembled

311 contigs for downstream analyses. False positives and false negatives among closely-related variants were mitigated

312 by the inclusion of the filtering steps described under Strategy 2. Across all 27 samples, the SAVAGE contigs are

$31398.3 \%$ and $99.7 \%$ accurate with respect to the expected MHC genotypes (Table 2).

314 While this analysis focused on genotyping using the same exon 2 reference sequences that are commonly utilized for

315 MiSeq amplicon analyses, the SAVAGE contigs are frequently much longer than these reference sequences (Figure

316 3). These extended contigs frequently contain exons 2 through 4 , plus the intervening introns, and could be used to

317 provide higher resolution genotyping than is possible using exon 2 sequence alone. Moreover, contigs that contain

318 complete sequences for exons 2-3 of MHC class I and exon 2 of MHC class II alleles meet the minimum criteria for

319 obtaining formal allele nomenclature for non-human primates from the IPD-MHC (Robinson et al. 2013). 


\section{Discussion}

323 Here we describe MES genotyping of Indian rhesus macaques. In our estimation, this method will supersede MiSeq

324 PCR amplicon genotyping as the most widely used macaque MHC genotyping assay in the future. This

325 methodology has a number of compelling advantages. Most importantly, exome sequencing dramatically improves

326 the overall quantity and quality of genomic information obtained from each sample. The same datasets used for

327 MHC analyses may be used to evaluate protein-coding genetic variation throughout the genome. The loss of start

328 and stop codons exome-wide can be obtained from the same datasets by modifying the workflows in silico, as

329 opposed to requiring the sequencing of multiple new sets of target genes (Yang et al. 2013). Exome-wide datasets

330 offer the promise of retrospectively identifying candidate DNA sequence variants that may be responsible for

331 unexpected experimental outcomes in studies with macaques and other nonhuman primates. The availability of

332 exome-wide sequences in conjunction with MHC genotypes may also increase the rigor of prospective macaque

333 experiments by enabling more sophisticated balancing of experimental groups, and exclusion of animals whose

334 genetics are likely to strongly bias experimental results (Loffredo et al. 2007; Reynolds et al. 2011 ; Haus et al.

335 2014).

336 These same exome datasets also offer the potential to improve the quality of MHC genotyping. Full-length long-read

337 MHC transcript sequencing offers exquisite resolution, but this technology can be labor intensive and difficult to

338 scale (Karl et al. 2017; Semler et al. 2018). The MiSeq exon 2 amplicon approach is limited in its allelic resolution,

339 but it is the current standard deep sequencing approach for high-throughput MHC genotyping in macaques. In this

340 report, we compare two novel strategies for MHC genotyping from MES datasets to this standard MiSeq amplicon

341 genotyping method. The results that we obtained with all three approaches show strong concordance with expected

342 MHC genotypes for the Indian rhesus macaques that were evaluated. As illustrated in Figure 3a, MHC class I

343 genomic contigs with an average length of approximately $1.1 \mathrm{~kb}$ can be assembled from sequence reads that were

344 initially extracted from the whole exome datasets.

345 Following the initial enrichment step, MHC class II genomic contigs averaging approximately 1.9 kb in length could

346 be assembled from whole exome sequence reads that mapped to $H L A-D R B 1,-D R B 4,-D R B 5,-D Q A 1,-D Q B 1,-$

$347 \quad D P A 1$ and $-D P B 1$ reference sequences, which contained exons $2-4$, and the pairs of introns (Figure $3 \mathbf{b})$. The 
addition of HLA spike-in probes containing both exon and intron sequences for the target capture step, and the introduction of $151 \mathrm{bp}$ paired end reads for the Illumina NovaSeq platform greatly facilitated our ability to assemble these extended MHC genomic contigs. Additional technological advances, including even longer sequence reads and more efficient assembly algorithms, will undoubtedly increase genomic contig lengths as well as MHC allelic resolution in future studies.

Both MHC genotyping approaches described here depend upon mapping exome sequence reads against a reference database of MHC class I and class II allele sequences. Currently, the IPD database essentially is restricted to coding regions for macaque MHC sequences, and many IPD entries are only partial transcript sequences that lack complete coding regions. In addition, it is very challenging to correctly phase short Illumina sequence read that map to different exons of a specific allelic variant and are separated by intronic sequences that span hundreds to thousands of base pairs in genomic DNA. Our results with the SAVAGE workflow (Figure 3) demonstrate that exome sequence reads that have been enriched with the enhanced MHC probe design described here can be assembled into genomic contigs that span multiple exons and introns. These contigs, therefore, could also be used to improve MHC reference databases, though this requires a major effort with more animals, and is beyond the scope of this

362 manuscript.

The current cost of genotyping is relatively high compared to amplicon deep sequencing, largely due to two

364 expenses. First, the amount of sequence data needed for a single sample can be high. Compared to conventional

365 MHC genotyping, where 192 macaques' data can be collected on a single instrument run, exome data acquisition is much more expensive. On an Illumina Novaseq instrument, which has a much higher run cost than the MiSeq, only

36770 exome samples can be sequenced simultaneously per lane of a S4 flow cell. However, this cost of sequencing is 368 rapidly decreasing, and as of early 2019, commercial providers have advertised sequencing for \$9 USD per Gb of 369 whole genome sequence data ${ }^{1}$. Second, a major expense in MHC genotyping is the production, validation, and use 370 of target-capture arrays, as well as the development of in silico data analysis workflows. The approaches described 371 here, in particular MHC genotyping from SAVAGE contigs produced by de novo assembly of exome reads, is 372 flexible and should be adaptable as sequencing approaches evolve and improve.

\footnotetext{
${ }^{1}$ Comment via Twitter 03/18/2019@albertvilella https://twitter.com/AlbertVilella/status/1107524501645000705
} 
373 A major goal for future studies will be to attempt to extend these contigs to encompass full length genomic MHC

374 sequences using SAVAGE or other assembly software tools. The relatively compact genomic structure and

375 consistent length of MHC class I genes increase the attainability of this goal. Establishment of comprehensive

376 macaque MHC allele databases of extended genomic sequences will greatly facilitate mapping of exome sequence

377 reads since they will be contiguous with the reference sequences instead of being interrupted by intervening

378 sequences between each exon that are not included in current non-human primate IPD-MHC databases (Maccari et

379 al. 2017).

380 These results demonstrate that MHC genotypes can be obtained by analyzing genomic DNA selectively enriched for

381 MHC and protein-coding gene sequences. This represents an important advance for characterizing MHC genetics in

382 macaques, and this suggests that analyses of whole exome and whole genome data will become the predominant

383 method for studying macaque genetics in the coming decade. 


\section{Acknowledgements}

386 We gratefully acknowledge Michele Di Mascio and his group at the National Institute of Allergy and Infectious

387 Diseases of the National Institutes of Health for providing rhesus macaque samples used in this study. We also

388 gratefully thank Brian Bushnell for assistance with the BBTools software, and the WNPRC for providing samples

389 from five related macaques.

390 This research was supported by contracts HHSN272201600007C from the National Institute of Allergy and

391 Infectious Diseases of the National Institutes of Health. This work was also supported in part by the Office of

392 Research Infrastructure Programs/OD (P51OD011106) awarded to the Wisconsin National Primate Research Center

393 at the University of Wisconsin-Madison. This research was also supported in part by grant R24-OD011173 from the

394 National Institutes of Health. This research was conducted in part at a facility constructed with support from

395 Research Facilities Improvement Program grants RR15459-01 and RR020141-01. 


\section{$405 \quad$ Figure Legends}

406

407

408

409

410

411

412

413

414

415

416

417

418

Table 1. Fraction of total exome sequence reads corresponding to exons 2 - 3 of MHC class I and exons 2 - 4 of MHC class II genes.

Table 2. Summary of discordant results for each MHC genotyping method by animal.

Figure 1. Comparison of MHC class I results from MiSeq PCR amplicon versus whole exome genotyping strategies for a representative breeding group of rhesus macaques. Results for each of the three methods are provided side-byside in the columns for each macaque. Each row indicates the detection of a specific MHC class I allele or lineage group of closely related sequences that are ambiguous because they are identical over the IPD exon 2 database sequence. Values in the body of this figure indicate the number of sequence reads supporting each allele call for the MiSeq and DSR methods while alleles supported by a SAVAGE contig are reported with a “1”. Discrepancies between the MiSeq (pink), DSR (yellow) or SAVAGE (blue) methods are highlighted by filled cells with borders.

Figure 2. Comparison of MHC class II results from MiSeq PCR amplicon versus whole exome genotyping strategies for a representative breeding group of rhesus macaques. Results for each of the three methods are provided side-by-side in the columns for these five related macaques. Each row indicates detection of a specific MHC class II allele or lineage group of closely related sequences that are ambiguous because they are identical over the IPD exon 2 database sequence. Values in the body of this figure indicate the number of sequence reads supporting each allele call for the MiSeq and DSR methods while alleles supported by a SAVAGE contig are reported with a "1". The Mamu-DPAI*11:01 allele missed by the MiSeq assay due to multiple mismatches versus the amplification primers is highlighted in pink.

Figure 3. Lengths of MHC sequence contigs assembled by the SAVAGE method for a representative breeding group of rhesus macaques. Values in the body of this figure indicate contig lengths generated for these MHC sequences in each animal. (A) MHC class I genotyping results are illustrated for the five animals in this breeding group. Three false negative allele calls for SAVAGE versus the expected genotypes for these animals are highlighted in magenta, e.g., Мamu-B*030g1 in Sire r07010. Sequences highlighted in red are associated with the maternal MHC Haplotype a that was inherited by Progeny 1 from its Dam. Sequences in Progeny 1 and Progeny 3 
430 for their MHC Haplotype b that was inherited from Sire r07010 are highlighted in dark blue while the light blue

431 sequences represent the alternate paternal Haplotype $\mathrm{c}$ that was inherited by Progeny 2 . Three unique extended

432 MHC Haplotypes in this breeding group are indicated with shades of grey (Haplotypes d - f). MHC allele groups

433 that are shared by both parental haplotypes are indicated by colored borders around filled cells. (B) MHC class II

434 genotyping results are illustrated for this same breeding group. (C) Abbreviated Mamu haplotype designations (Karl

435 et al. 2013) are summarized for the six extended MHC haplotypes identified by segregation in this breeding group.

436 For example, the twenty MHC sequences (red) that are associated with extended Haplotype a can be summarized by

437 the following string of abbreviated Mamu haplotype designations: Mamu-

438 A004/B048/DR04a/DQA01g1/DQB06:01/DPA02g1/DPB15g. 


\section{Supplementary Materials}

442 Supplementary Table 1. Fraction of total exome sequence reads corresponding to MHC class I and class II genes

443 after target capture with the VCRome2.1 probe design alone. This exome sequence dataset was described previously

444 by Ericsen and coworkers (Ericsen et al. 2014).

445 Supplementary Figure 1. Comparison of MHC class I results from MiSeq PCR amplicon versus whole exome

446 genotyping assays with the DSR and SAVAGE strategies for all 27 animals. Results for each of the three method are 447 provided side-by-side in the columns for each macaque. Each row indicates the detection of a specific MHC class I

448 allele or lineage group of closely related sequences that are ambiguous because they are identical over the IPD exon

4492 database sequence. Values in the body of this figure indicate the number of sequence reads supporting each allele

450 call for the MiSeq and DSR methods while alleles supported by a SAVAGE contig are reported with a “1”.

451 Discrepancies between the MiSeq (pink), DSR (yellow) or SAVAGE (blue) methods are highlighted by filled cells 452 with borders. 


\section{References}

Animal Welfare Act. ‘The Animal Welfare Act - Public Law 89-544 Act of August 24, 1966. 1966. https://www.nal.usda.gov/awic/animal-welfare-act-public-law-89-544-act-august-24-1966 . Accessed 05/01/2019

Baaijens, J. A., A. Z. E. Aabidine, E. Rivals, and A. Schonhuth. 2017. De novo assembly of viral quasispecies using overlap graphs. Genome Res 27: 835-848. https://www.ncbi.nlm.nih.gov/pubmed/28396522

Bimber, B. N., R. Ramakrishnan, R. Cervera-Juanes, R. Madhira, S. M. Peterson, R. B. J. Norgren, and B. Ferguson. 2017. Whole genome sequencing predicts novel human disease models in rhesus macaques. Genomics 109: 214-220. https://www.ncbi.nlm.nih.gov/pubmed/28438488

Brown, J. H., T. S. Jardetzky, J. C. Gorga, L. J. Stern, R. G. Urban, J. L. Strominger, and D. C. Wiley. 1993. Threedimensional structure of the human class II histocompatibility antigen HLA-DR1. Nature 364: 33-39. https://www.ncbi.nlm.nih.gov/pubmed/8316295

Bushnell, B., J. Rood, and E. Singer. 2017. BBMerge - Accurate paired shotgun read merging via overlap. PLoS One 12: e0185056. https://www.ncbi.nlm.nih.gov/pubmed/29073143

Cao, H., J. Wu, Y. Wang, H. Jiang, T. Zhang, X. Liu, Y. Xu, D. Liang, P. Gao, Y. Sun, B. Gifford, M. D’Ascenzo, X. Liu, L. C. Tellier, F. Yang, X. Tong, D. Chen, J. Zheng, W. Li, T. Richmond, X. Xu, J. Wang, and Y. Li. 2013. An integrated tool to study MHC region: accurate SNV detection and HLA genes typing in human MHC region using targeted high-throughput sequencing. PLoS One 8: e69388. https://www.ncbi.nlm.nih.gov/pubmed/23894464

Clark, M. J., R. Chen, and M. Snyder. 2013. Exome sequencing by targeted enrichment. Curr Protoc Mol Biol Chapter 7: Unit7.12. https://www.ncbi.nlm.nih.gov/pubmed/23547016

Cornish, A. S., R. M. Gibbs, and R. B. J. Norgren. 2016. Exome screening to identify loss-of-function mutations in the rhesus macaque for development of preclinical models of human disease. BMC Genomics 17: 170. https://www.ncbi.nlm.nih.gov/pubmed/26935327

Daza-Vamenta, R., G. Glusman, L. Rowen, B. Guthrie, and D. E. Geraghty. 2004. Genetic divergence of the rhesus macaque major histocompatibility complex. Genome Res 14: 1501-1515. https://www.ncbi.nlm.nih.gov/pubmed/15289473 
de Manuel, M., T. Shiina, S. Suzuki, N. Dereuddre-Bosquet, H. J. Garchon, M. Tanaka, N. Congy-Jolivet, A. Aarnink, R. Le Grand, T. Marques-Bonet, and A. Blancher. 2018. Whole genome sequencing in the search for genes associated with the control of SIV infection in the Mauritian macaque model. Sci Rep 8: 7131. https://www.ncbi.nlm.nih.gov/pubmed/29739964

Edgar, R. C. 2010. Search and clustering orders of magnitude faster than BLAST. Bioinformatics 26: $2460-2461$. https://www.ncbi.nlm.nih.gov/pubmed/20709691

Ericsen, A. J., G. J. Starrett, J. M. Greene, M. Lauck, M. Raveendran, D. R. Deiros, M. S. Mohns, N. Vince, B. T. Cain, N. H. Pham, J. T. Weinfurter, A. L. Bailey, M. L. Budde, R. W. Wiseman, R. Gibbs, D. Muzny, T. C. Friedrich, J. Rogers, and D. H. O’Connor. 2014. Whole genome sequencing of SIV-infected macaques identifies candidate loci that may contribute to host control of virus replication. Genome Biol 15: 478. https://www.ncbi.nlm.nih.gov/pubmed/25418588

Garcia, K. C., and E. J. Adams. 2005. How the T cell receptor sees antigen--a structural view. Cell 122: $333-336$. https://www.ncbi.nlm.nih.gov/pubmed/16096054

Haus, T., B. Ferguson, J. Rogers, G. Doxiadis, U. Certa, N. J. Rose, R. Teepe, G. F. Weinbauer, and C. Roos. 2014. Genome typing of nonhuman primate models: implications for biomedical research. Trends Genet 30: 482487. https://www.ncbi.nlm.nih.gov/pubmed/24954183

Horton, R., R. Gibson, P. Coggill, M. Miretti, R. J. Allcock, J. Almeida, S. Forbes, J. G. Gilbert, K. Halls, J. L. Harrow, E. Hart, K. Howe, D. K. Jackson, S. Palmer, A. N. Roberts, S. Sims, C. A. Stewart, J. A. Traherne, S. Trevanion, L. Wilming, J. Rogers, P. J. de Jong, J. F. Elliott, S. Sawcer, J. A. Todd, J. Trowsdale, and S. Beck. 2008. Variation analysis and gene annotation of eight MHC haplotypes: the MHC Haplotype Project. Immunogenetics 60: 1-18. https://www.ncbi.nlm.nih.gov/pubmed/18193213

Kaizu, M., G. J. Borchardt, C. E. Glidden, D. L. Fisk, J. T. Loffredo, D. I. Watkins, and W. M. Rehrauer. 2007. Molecular typing of major histocompatibility complex class I alleles in the Indian rhesus macaque which restrict SIV CD8+ T cell epitopes. Immunogenetics 59: 693-703. https://www.ncbi.nlm.nih.gov/pubmed/17641886

Karl, J. A., P. S. Bohn, R. W. Wiseman, F. A. Nimityongskul, S. M. Lank, G. J. Starrett, and D. H. O’Connor. 2013. Major histocompatibility complex class I haplotype diversity in Chinese rhesus macaques. G3 (Bethesda) 3 : 1195-1201. https://www.ncbi.nlm.nih.gov/pubmed/23696100 
Karl, J. A., M. E. Graham, R. W. Wiseman, K. E. Heimbruch, S. M. Gieger, G. G. Doxiadis, R. E. Bontrop, and D. H. O'Connor. 2017. Major histocompatibility complex haplotyping and long-amplicon allele discovery in cynomolgus macaques from Chinese breeding facilities. Immunogenetics 69: 211-229. https://www.ncbi.nlm.nih.gov/pubmed/28078358

Karl, J. A., K. E. Heimbruch, C. E. Vriezen, C. J. Mironczuk, D. M. Dudley, R. W. Wiseman, and D. H. O’Connor. 2014. Survey of major histocompatibility complex class II diversity in pig-tailed macaques. Immunogenetics 66: 613-623. https://www.ncbi.nlm.nih.gov/pubmed/25129472

Kishikawa, T., Y. Momozawa, T. Ozeki, T. Mushiroda, H. Inohara, Y. Kamatani, M. Kubo, and Y. Okada. 2019. Empirical evaluation of variant calling accuracy using ultra-deep whole-genome sequencing data. Sci Rep 9: 1784. https://www.ncbi.nlm.nih.gov/pubmed/30741997

Koster, J., and S. Rahmann. 2012. Snakemake--a scalable bioinformatics workflow engine. Bioinformatics 28: 25202522. https://www.ncbi.nlm.nih.gov/pubmed/22908215

Loffredo, J. T., J. Maxwell, Y. Qi, C. E. Glidden, G. J. Borchardt, T. Soma, A. T. Bean, D. R. Beal, N. A. Wilson, W. M. Rehrauer, J. D. Lifson, M. Carrington, and D. I. Watkins. 2007. Mamu-B*08-positive macaques control simian immunodeficiency virus replication. J Virol 81: 8827-8832. https://www.ncbi.nlm.nih.gov/pubmed/17537848

Loffredo, J. T., J. Sidney, A. T. Bean, D. R. Beal, W. Bardet, A. Wahl, O. E. Hawkins, S. Piaskowski, N. A. Wilson, W. H. Hildebrand, D. I. Watkins, and A. Sette. 2009. Two MHC class I molecules associated with elite control of immunodeficiency virus replication, Mamu-B*08 and HLA-B*2705, bind peptides with sequence similarity. J Immunol 182: 7763-7775. https://www.ncbi.nlm.nih.gov/pubmed/19494300

Maccari, G., J. Robinson, K. Ballingall, L. A. Guethlein, U. Grimholt, J. Kaufman, C. S. Ho, N. G. de Groot, P. Flicek, R. E. Bontrop, J. A. Hammond, and S. G. Marsh. 2017. IPD-MHC 2.0: an improved inter-species database for the study of the major histocompatibility complex. Nucleic Acids Res 45: D860-D864. https://www.ncbi.nlm.nih.gov/pubmed/27899604

Malissen, M., B. Malissen, and B. R. Jordan. 1982. Exon/intron organization and complete nucleotide sequence of an HLA gene. Proc Natl Acad Sci U S A 79: 893-897. https://www.ncbi.nlm.nih.gov/pubmed/6461010

Mothe, B. R., J. Weinfurter, C. Wang, W. Rehrauer, N. Wilson, T. M. Allen, D. B. Allison, and D. I. Watkins. 2003. Expression of the major histocompatibility complex class I molecule Mamu-A*01 is associated with control 
of simian immunodeficiency virus SIVmac239 replication. J Virol 77: 2736-2740. https://www.ncbi.nlm.nih.gov/pubmed/12552014

Muhl, T., M. Krawczak, P. Ten Haaft, G. Hunsmann, and U. Sauermann. 2002. MHC class I alleles influence setpoint viral load and survival time in simian immunodeficiency virus-infected rhesus monkeys. J Immunol 169: 3438-3446. https://www.ncbi.nlm.nih.gov/pubmed/12218167

Nomura, T., H. Yamamoto, T. Shiino, N. Takahashi, T. Nakane, N. Iwamoto, H. Ishii, T. Tsukamoto, M. Kawada, S. Matsuoka, A. Takeda, K. Terahara, Y. Tsunetsugu-Yokota, N. Iwata-Yoshikawa, H. Hasegawa, T. Sata, T. K. Naruse, A. Kimura, and T. Matano. 2012. Association of major histocompatibility complex class I haplotypes with disease progression after simian immunodeficiency virus challenge in burmese rhesus macaques. J Virol 86: 6481-6490. https://www.ncbi.nlm.nih.gov/pubmed/22491464

Otting, N., M. K. van der Wiel, N. de Groot, A. J. de Vos-Rouweler, N. G. de Groot, G. G. Doxiadis, R. W. Wiseman, D. H. O'Connor, and R. E. Bontrop. 2017. The orthologs of HLA-DQ and -DP genes display abundant levels of variability in macaque species. Immunogenetics 69: 87-99. https://www.ncbi.nlm.nih.gov/pubmed/27771735

Posey, J. E., J. A. Rosenfeld, R. A. James, M. Bainbridge, Z. Niu, X. Wang, S. Dhar, W. Wiszniewski, Z. H. Akdemir, T. Gambin, F. Xia, R. E. Person, M. Walkiewicz, C. A. Shaw, V. R. Sutton, A. L. Beaudet, D. Muzny, C. M. Eng, Y. Yang, R. A. Gibbs, J. R. Lupski, E. Boerwinkle, and S. E. Plon. 2016. Molecular diagnostic experience of whole-exome sequencing in adult patients. Genet Med 18: 678-685. https://www.ncbi.nlm.nih.gov/pubmed/26633545

Prall, T. M., M. E. Graham, J. A. Karl, R. W. Wiseman, A. J. Ericsen, M. Raveendran, R. Alan Harris, D. M. Muzny, R. A. Gibbs, J. Rogers, and D. H. O'Connor. 2017. Improved full-length killer cell immunoglobulinlike receptor transcript discovery in Mauritian cynomolgus macaques. Immunogenetics 69: 325-339. https://www.ncbi.nlm.nih.gov/pubmed/28343239

Reynolds, M. R., J. B. Sacha, A. M. Weiler, G. J. Borchardt, C. E. Glidden, N. C. Sheppard, F. A. Norante, P. A. Castrovinci, J. J. Harris, H. T. Robertson, T. C. Friedrich, A. B. McDermott, N. A. Wilson, D. B. Allison, W. C. Koff, W. E. Johnson, and D. I. Watkins. 2011. The TRIM5 \{alpha\} genotype of rhesus macaques affects acquisition of simian immunodeficiency virus SIVsmE660 infection after repeated limiting-dose intrarectal challenge. J Virol 85: 9637-9640. https://www.ncbi.nlm.nih.gov/pubmed/21734037 
Robinson, J., J. A. Halliwell, H. McWilliam, R. Lopez, and S. G. Marsh. 2013. IPD--the Immuno Polymorphism Database. Nucleic Acids Res 41: D1234-40. https://www.ncbi.nlm.nih.gov/pubmed/23180793

Semler, M. R., R. W. Wiseman, J. A. Karl, M. E. Graham, S. M. Gieger, and D. H. O’Connor. 2018. Novel fulllength major histocompatibility complex class I allele discovery and haplotype definition in pig-tailed macaques. Immunogenetics 70: 381-399. https:/www.ncbi.nlm.nih.gov/pubmed/29134258

Shiina, T., A. Blancher, H. Inoko, and J. K. Kulski. 2017. Comparative genomics of the human, macaque and mouse major histocompatibility complex. Immunology 150: 127-138. https://www.ncbi.nlm.nih.gov/pubmed/27395034

Silver, Z. A., and D. I. Watkins. 2017. The role of MHC class I gene products in SIV infection of macaques. Immunogenetics 69: 511-519. https://www.ncbi.nlm.nih.gov/pubmed/28695289

Vallender, E. J. 2011. Expanding whole exome resequencing into non-human primates. Genome Biol 12 : R87. https://www.ncbi.nlm.nih.gov/pubmed/21917143

Weatherall, D. 2006. The use of non-human primates in research. 147. https://mrc.ukri.org/documents/pdf/the-useof-non-human-primates-in-research/ . Accessed 04/30/2019

Williams, T. M. 2001. Human leukocyte antigen gene polymorphism and the histocompatibility laboratory. $J$ Mol Diagn 3: 98-104. https://www.ncbi.nlm.nih.gov/pubmed/11486048

Wiseman, R. W., J. A. Karl, B. N. Bimber, C. E. O’Leary, S. M. Lank, J. J. Tuscher, A. M. Detmer, P. Bouffard, N. Levenkova, C. L. Turcotte, E. J. Szekeres, C. Wright, T. Harkins, and D. H. O’Connor. 2009. Major histocompatibility complex genotyping with massively parallel pyrosequencing. Nat Med 15: 1322-1326. https://www.ncbi.nlm.nih.gov/pubmed/19820716

Wiseman, R. W., J. A. Karl, P. S. Bohn, F. A. Nimityongskul, G. J. Starrett, and D. H. O’Connor. 2013. Haplessly hoping: macaque major histocompatibility complex made easy. ILAR J 54: 196-210. https://www.ncbi.nlm.nih.gov/pubmed/24174442

Wittig, M., J. A. Anmarkrud, J. C. Kassens, S. Koch, M. Forster, E. Ellinghaus, J. R. Hov, S. Sauer, M. Schimmler, M. Ziemann, S. Gorg, F. Jacob, T. H. Karlsen, and A. Franke. 2015. Development of a high-resolution NGSbased HLA-typing and analysis pipeline. Nucleic Acids Res 43: e70. https://www.ncbi.nlm.nih.gov/pubmed/25753671 
Xie, C., Z. X. Yeo, M. Wong, J. Piper, T. Long, E. F. Kirkness, W. H. Biggs, K. Bloom, S. Spellman, C. VierraGreen, C. Brady, R. H. Scheuermann, A. Telenti, S. Howard, S. Brewerton, Y. Turpaz, and J. C. Venter. 2017. Fast and accurate HLA typing from short-read next-generation sequence data with xHLA. Proc Natl Acad Sci U S A 114: 8059-8064. https://www.ncbi.nlm.nih.gov/pubmed/28674023

Xue, C., M. Raveendran, R. A. Harris, G. L. Fawcett, X. Liu, S. White, M. Dahdouli, D. Rio Deiros, J. E. Below, W. Salerno, L. Cox, G. Fan, B. Ferguson, J. Horvath, Z. Johnson, S. Kanthaswamy, H. M. Kubisch, D. Liu, M. Platt, D. G. Smith, B. Sun, E. J. Vallender, F. Wang, R. W. Wiseman, R. Chen, D. M. Muzny, R. A. Gibbs, F. Yu, and J. Rogers. 2016. The population genomics of rhesus macaques (Macaca mulatta) based on wholegenome sequences. Genome Res 26: 1651-1662. https://www.ncbi.nlm.nih.gov/pubmed/27934697

Yang, Y., D. M. Muzny, J. G. Reid, M. N. Bainbridge, A. Willis, P. A. Ward, A. Braxton, J. Beuten, F. Xia, Z. Niu, M. Hardison, R. Person, M. R. Bekheirnia, M. S. Leduc, A. Kirby, P. Pham, J. Scull, M. Wang, Y. Ding, S. E. Plon, J. R. Lupski, A. L. Beaudet, R. A. Gibbs, and C. M. Eng. 2013. Clinical whole-exome sequencing for the diagnosis of mendelian disorders. N Engl J Med 369: 1502-1511. https://www.ncbi.nlm.nih.gov/pubmed/24088041

Yang, Y., D. M. Muzny, F. Xia, Z. Niu, R. Person, Y. Ding, P. Ward, A. Braxton, M. Wang, C. Buhay, N. Veeraraghavan, A. Hawes, T. Chiang, M. Leduc, J. Beuten, J. Zhang, W. He, J. Scull, A. Willis, M. Landsverk, W. J. Craigen, M. R. Bekheirnia, A. Stray-Pedersen, P. Liu, S. Wen, W. Alcaraz, H. Cui, M. Walkiewicz, J. Reid, M. Bainbridge, A. Patel, E. Boerwinkle, A. L. Beaudet, J. R. Lupski, S. E. Plon, R. A. Gibbs, and C. M. Eng. 2014. Molecular findings among patients referred for clinical whole-exome sequencing. JAMA 312: 1870-1879. https://www.ncbi.nlm.nih.gov/pubmed/25326635

Zimin, A. V., A. S. Cornish, M. D. Maudhoo, R. M. Gibbs, X. Zhang, S. Pandey, D. T. Meehan, K. Wipfler, S. E. Bosinger, Z. P. Johnson, G. K. Tharp, G. Marcais, M. Roberts, B. Ferguson, H. S. Fox, T. Treangen, S. L. Salzberg, J. A. Yorke, and R. B. J. Norgren. 2014. A new rhesus macaque assembly and annotation for nextgeneration sequencing analyses. Biol Direct 9: 20. https://www.ncbi.nlm.nih.gov/pubmed/25319552 
bioRxiv preprint doi: https://doi.org/10.1101/625244; this version posted May 2, 2019. The copyright holder for this preprint (which was not certified by peer review) is the author/funder. All rights reserved. No reuse allowed without permission. 
bioRxiv preprint doi: https://doi.org/10.1101/625244; this version posted May 2, 2019. The copyright holder for this preprint (which was not certified by peer review) is the author/funder. All rights reserved. No reuse allowed without permission.

Table 1. Fraction of total exome sequence reads corresponding to exons 2 - 3 of MHC class I and exons 2 - 4 of MHC class II genes

\begin{tabular}{|c|c|c|c|c|c|c|c|c|c|c|}
\hline Animal ID & SRA Accession & $\begin{array}{l}\text { Total Raw } \\
\text { Reads }\end{array}$ & $\begin{array}{c}\text { MHC-I } \\
\text { Reads } \\
\text { Extracted }\end{array}$ & $\begin{array}{c}\text { DRB1/3/4/5 } \\
\text { Reads } \\
\text { Extracted }\end{array}$ & $\begin{array}{c}\text { DQA1 } \\
\text { Reads } \\
\text { Extracted }\end{array}$ & $\begin{array}{c}\text { DQB1 } \\
\text { Reads } \\
\text { Extracted }\end{array}$ & $\begin{array}{c}\text { DPA1 } \\
\text { Reads } \\
\text { Extracted }\end{array}$ & $\begin{array}{c}\text { DPB1 } \\
\text { Reads } \\
\text { Extracted }\end{array}$ & $\begin{array}{c}\text { MHC } \\
\text { Reads } \\
\text { Extracted }\end{array}$ & $\begin{array}{r}\text { MHC \% } \\
\text { of total }\end{array}$ \\
\hline r05029 & SAMN11131055 & $87,345,430$ & 79,712 & 215,654 & 24,266 & 39,188 & 29,604 & 112,376 & 500,800 & 0.57 \\
\hline r17099 & SAMN11131056 & $67,632,236$ & 32,406 & 89,946 & 9,694 & 16,782 & 11,214 & 47,974 & 208,016 & 0.31 \\
\hline r07010 & SAMN11173514 & $81,062,856$ & 71,694 & 204,352 & 22,528 & 38,382 & 25,392 & 111,626 & 473,974 & 0.58 \\
\hline r17041 & SAMN11131058 & $80,004,430$ & 30,852 & 244,326 & 9,746 & 16,310 & 12,644 & 151,376 & 465,254 & 0.58 \\
\hline r17061 & SAMN11131059 & $78,428,820$ & 70,082 & 204,694 & 20,776 & 41,018 & 19,440 & 95,538 & 451,548 & 0.58 \\
\hline $\mathrm{J} 8 \mathrm{R}$ & SAMN11282383 & $80,868,782$ & 45,164 & 87,864 & 11,878 & 19,736 & 13,810 & 57,146 & 235,598 & 0.29 \\
\hline J01 & SAMN11282382 & $61,009,650$ & 34,342 & 87,124 & 9,050 & 16,628 & 10,958 & 46,964 & 205,066 & 0.34 \\
\hline ZC08 & SAMN11282384 & $60,693,394$ & 31,168 & 81,586 & 10,392 & 15,660 & 10,172 & 45,748 & 194,726 & 0.32 \\
\hline DGKG & SAMN11282378 & $80,530,772$ & 48,658 & 130,256 & 12,788 & 22,682 & 13,372 & 62,990 & 290,746 & 0.36 \\
\hline CF18 & SAMN11282368 & $68,593,710$ & 37,792 & 96,842 & 10,864 & 18,226 & 13,118 & 51,556 & 228,398 & 0.33 \\
\hline DE1AA & SAMN11282369 & $65,712,570$ & 39,334 & 108,002 & 10,060 & 17,124 & 11,840 & 51,134 & 237,494 & 0.36 \\
\hline DEG8 & SAMN11282370 & $68,490,344$ & 35,566 & 116,824 & 10,404 & 19,278 & 12,294 & 55,086 & 249,452 & 0.36 \\
\hline DEXX & SAMN11282371 & $67,451,626$ & 33,900 & 128,128 & 10,442 & 18,898 & 9,778 & 53,258 & 254,404 & 0.38 \\
\hline $\mathrm{DF} 24$ & SAMN11282372 & $67,768,724$ & 41,362 & 96,172 & 10,606 & 18,176 & 10,976 & 50,612 & 227,904 & 0.34 \\
\hline DF64 & SAMN11282373 & $65,762,818$ & 37,426 & 107,974 & 11,222 & 15,632 & 11,446 & 51,214 & 234,914 & 0.36 \\
\hline DF6T & SAMN11282374 & $71,472,222$ & 41,160 & 101,102 & 11,052 & 18,794 & 13,492 & 51,626 & 237,226 & 0.33 \\
\hline DFET & SAMN11282375 & $77,040,660$ & 49,068 & 128,862 & 12,588 & 20,952 & 12,586 & 58,238 & 282,294 & 0.37 \\
\hline DFV0 & SAMN11282377 & $73,877,184$ & 41,924 & 98,866 & 11,630 & 17,690 & 13,952 & 52,394 & 236,456 & 0.32 \\
\hline DJ94 & SAMN11282379 & $66,051,412$ & 39,616 & 88,196 & 9,910 & 15,892 & 10,978 & 45,972 & 210,564 & 0.32 \\
\hline HIH & SAMN11282380 & $76,491,578$ & 41,308 & 85,732 & 11,148 & 18,366 & 14,102 & 50,746 & 221,402 & 0.29 \\
\hline DFJT & SAMN11282376 & $62,249,064$ & 37,728 & 78,860 & 9,666 & 15,268 & 11,426 & 43,086 & 196,034 & 0.31 \\
\hline HLJ & SAMN11282381 & $68,288,558$ & 41,748 & 94,570 & 9,960 & 17,240 & 12,756 & 48,356 & 224,630 & 0.33 \\
\hline 37360 & SAMN11282363 & $62,310,824$ & 38,200 & 97,452 & 9,866 & 15,024 & 10,320 & 46,140 & 217,002 & 0.35 \\
\hline $0 \mathrm{L7}$ & SAMN11282364 & $64,145,834$ & 35,302 & 128,778 & 9,310 & 19,992 & 10,686 & 54,196 & 258,264 & 0.40 \\
\hline OR1 & SAMN11282365 & $64,789,208$ & 36,462 & 86,772 & 10,656 & 17,258 & 11,982 & 46,124 & 209,254 & 0.32 \\
\hline 0R8 & SAMN11282366 & $72,836,490$ & 38,286 & 118,184 & 10,500 & 22,248 & 13,170 & 56,142 & 258,530 & 0.35 \\
\hline \multirow[t]{2}{*}{ OTI } & SAMN11282367 & $80,730,750$ & 41,552 & 106,356 & 13,364 & 20,714 & 14,860 & 57,744 & 254,590 & 0.32 \\
\hline & Average & $\mathbf{7 0 , 5 4 9 , 7 8 9}$ & 42,660 & 119,018 & 12,014 & 20,487 & 13,569 & 61,310 & 269,057 & 0.37 \\
\hline
\end{tabular}




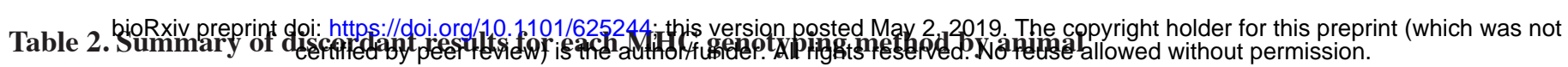

\begin{tabular}{|c|c|c|c|c|c|c|c|c|}
\hline \multirow[b]{2}{*}{ Animal ID } & \multicolumn{4}{|c|}{ MHC class I } & \multicolumn{4}{|c|}{ MHC class II } \\
\hline & $\begin{array}{l}\text { Total Alleles } \\
\text { Expected }\end{array}$ & $\begin{array}{c}\text { \# MiSeq } \\
\text { Discordance }\end{array}$ & $\begin{array}{c}\text { \# DSR } \\
\text { Discordance }\end{array}$ & $\begin{array}{l}\text { \# SAVAGE } \\
\text { Discordance }\end{array}$ & $\begin{array}{l}\text { Total Alleles } \\
\text { Expected }\end{array}$ & $\begin{array}{c}\text { \# MiSeq } \\
\text { Discordance }\end{array}$ & $\begin{array}{c}\text { \# DSR } \\
\text { Discordance }\end{array}$ & $\begin{array}{l}\text { \# SAVAGE } \\
\text { Discordance }\end{array}$ \\
\hline r05029 & 28 & 2 & 1 & & 10 & & & \\
\hline r17099 & 25 & 3 & & 1 & 12 & & & \\
\hline r07010 & 26 & 3 & 1 & 1 & 12 & & & \\
\hline r17041 & 31 & 3 & & 1 & 10 & & & \\
\hline r17061 & 29 & 1 & 2 & & 12 & 1 & & \\
\hline J8R & 29 & 4 & 1 & & 12 & 2 & & \\
\hline J01 & 30 & 3 & 1 & 1 & 11 & 1 & & \\
\hline ZC08 & 24 & 2 & & 1 & 12 & & & \\
\hline DGKG & 28 & 4 & 1 & & 13 & & & \\
\hline CF18 & 28 & 3 & & & 12 & & & \\
\hline DE1AA & 30 & 5 & 1 & & 13 & & & \\
\hline DEG8 & 27 & 3 & 1 & & 13 & & & \\
\hline DEXX & 24 & 3 & 1 & 1 & 14 & & & \\
\hline $\mathrm{DF} 24$ & 32 & 4 & & 1 & 12 & & & \\
\hline DF64 & 30 & 3 & 1 & 1 & 13 & & & \\
\hline DF6T & 31 & 2 & & 1 & 10 & & & \\
\hline DFET & 30 & 3 & 1 & 1 & 13 & 1 & & 1 \\
\hline DFV0 & 28 & 2 & & & 6 & & & \\
\hline DJ94 & 34 & 3 & 2 & 1 & 12 & & & \\
\hline $\mathrm{HIH}$ & 30 & 3 & & & 10 & & & \\
\hline DFJT & 28 & 2 & 3 & & 11 & & & \\
\hline HLJ & 29 & 2 & 1 & 1 & 11 & & & \\
\hline 37360 & 29 & 2 & 1 & 1 & 13 & 1 & & \\
\hline $0 \mathrm{~L} 7$ & 20 & 2 & & & 14 & & & \\
\hline 0R1 & 17 & 1 & & & 12 & & & \\
\hline 0R8 & 25 & 2 & & & 13 & 1 & & \\
\hline OTI & 25 & 2 & 1 & & 12 & & & \\
\hline Total & 747 & 72 & 20 & 13 & 318 & 7 & 0 & 1 \\
\hline Discordance (\%) & & 9.6 & 2.7 & 1.7 & & 2.2 & 0.0 & 0.3 \\
\hline Concordance (\%) & & 90.4 & 97.3 & 98.3 & & 97.8 & 100.0 & 99.7 \\
\hline
\end{tabular}




\begin{tabular}{|c|c|c|c|c|c|c|c|c|c|c|c|c|c|c|c|c|}
\hline \multirow[t]{2}{*}{$\begin{array}{l}\text { Relationship } \\
\text { Animal_ID }\end{array}$} & \multicolumn{3}{|c|}{$\underset{\text { r05029 }}{\text { Dam }}$} & \multicolumn{3}{|c|}{$\begin{array}{c}\text { Progeny } 1 \\
\text { r17099 }\end{array}$} & \multicolumn{3}{|c|}{$\begin{array}{c}\text { Sire } \\
\text { r07010 }\end{array}$} & \multicolumn{3}{|c|}{$\begin{array}{c}\text { Progeny } 2 \\
\text { r17041 }\end{array}$} & & $\begin{array}{l}\text { rogen } \\
\text { r1706 }\end{array}$ & & \\
\hline & MiSeq & DSR S & AVAGE & Miseq & DSR & AVAGE & Miseq & DSR & SAVAGE & MiSeq & DSR S & SAVAGE & MiSeq & DSR S & AVAGE & Ambiguous Allele Group \\
\hline Mamu-A Major Alleles & & & & & & & & & & & & & & & & \\
\hline Мати-AI*001g2 & & & & 168 & 27 & 1 & 158 & 62 & 1 & & & & 210 & 74 & 1 & $A 1 * 001: 01, A 1 * 001: 02$ \\
\hline Мати-AI*002:01 & 335 & 136 & 1 & & & & & & & & & & 447 & 127 & 1 & \\
\hline Мати-AI*004gl & 151 & 137 & 1 & 126 & 51 & 1 & & & & & & & & & & AI*004_6_alleles \\
\hline Мати-AI*008g1 & & & & & & & 306 & 79 & 1 & 352 & 36 & 1 & & & & $A 1 * 008 \_5 \_$alleles \\
\hline Мати-AI*023_A1*106gI & & & & & & & & & & 228 & 36 & 1 & & & & $A 1 * 023: 01, A 1 * 023: 02, A 1 * 106: 01$ \\
\hline Мати-A1*059:01 & & 45 & & & & & & & & & & & & & & \\
\hline Mamu-A Minor Alleles & & & & & & & & & & & & & & & & \\
\hline Мати-A2*05g1 & & & & 319 & 25 & 1 & 712 & 162 & 1 & 423 & 15 & 1 & 438 & 56 & 1 & A2*05_32_alleles \\
\hline Мати-A3*13_A4*I4gI & 77 & 79 & 1 & & & & & & & & & & 91 & 88 & 1 & A3*13_7_alleles, $A 4 * 14 \_1$ _allele \\
\hline Мати-A3*13gI & & & & & & & 93 & 85 & 1 & 97 & 31 & 1 & & & & $A 3 * 13: 03, A 3 * 13: 11$ \\
\hline Мати-A4*I4gI & 145 & 104 & 1 & 164 & 23 & 1 & & & & 199 & 29 & 1 & & & & A4*14_14_alleles \\
\hline Mamu-E Alleles & & & & & & & & & & & & & & & & \\
\hline Мати-E*02g1 & 775 & 185 & 1 & 1923 & 169 & 1 & 2041 & 303 & 1 & 3240 & 125 & 1 & 914 & 120 & 1 & $E^{*} 02 \_9 \_$alleles \\
\hline Мати- $E^{*} 02 g 4$ & 307 & 106 & 1 & & & & & & & & & & 382 & 95 & 1 & $E^{*} 02: 13, E^{*} 02: 21, E^{*} 02: 29$ \\
\hline Мати- $E^{*} 02 g 5$ & 1104 & 134 & 1 & & & & & & & & & & 1583 & 69 & 1 & $E^{*} 02: 04, E^{*} 02: 06$ \\
\hline Мати- $E^{*} 02 g 7$ & & & & & & & 467 & 152 & 1 & 634 & 50 & 1 & & & & $E^{*} 02: 12: 01, E^{*} 02: 28$ \\
\hline Мати-E* $02 g 8$ & 689 & 116 & 1 & 1914 & 92 & 1 & 781 & 85 & 1 & & & & 710 & 85 & 1 & $E^{*} 02: 20, E^{*} 02: 30$ \\
\hline Mamu-B Major Alleles & & & & & & & & & & & & & & & & \\
\hline Мати- $B^{*} 00 \lg I$ & & & & & & & 161 & 60 & 1 & 143 & 28 & 1 & & & & $B^{*} 001 \_a l l e l e s$ \\
\hline Мати- $B^{*} 007: 07$ & & & & & & & 25 & & & 27 & & & & & & \\
\hline Мати- $B * 007 g 1$ & & & & & & & 161 & 33 & 1 & 132 & 12 & 1 & & & & $B^{*} 007 \quad 6$ alleles \\
\hline Мати- $B * 012: 01$ & & & & & & & & & & & & & 284 & 60 & 1 & \\
\hline Мати-B_022gI & & & & & & & & & & & & & 171 & 46 & 1 & $B^{*} 022: 01, B^{*} 022: 02$ \\
\hline Мати- $B^{*} 027 g 1$ & & & & & & & & & & 93 & 32 & 1 & & & & $B * 027: 03, B * 027: 04$ \\
\hline Мати- $B^{*} 030 \mathrm{~g} 1$ & & & & 270 & 12 & 1 & 303 & 73 & - & 318 & 11 & 1 & 671 & 103 & 1 & $B * 030 \_6 \_a l l e l e s$ \\
\hline Мати- $B^{*} 030 g 2$ & & & & & & & 306 & 79 & 1 & 306 & 29 & 1 & & & & $B * 030: 02, B * 030: 05$ \\
\hline Мати- $B * 031: 03$ & & & & 105 & 12 & 1 & 139 & 26 & 1 & & & & 198 & 35 & 1 & \\
\hline Мати- $B * 03 \lg 1$ & & & & & & & & & & & & & 127 & 40 & 1 & $B * 031: 01, B * 031: 02$ \\
\hline Мати- $B^{*} 041 \mathrm{~g} I$ & 128 & 47 & 1 & 57 & 24 & 1 & & & & & & & & & & $B^{* 041: 01, B * 041: 02}$ \\
\hline Мати- $B * 043: 01$ & & & & 223 & 18 & 1 & 224 & 49 & 1 & 263 & 16 & 1 & 220 & 41 & 1 & \\
\hline Мати- $B^{*} 048: 01$ & 275 & 91 & 1 & 318 & 26 & 1 & & & & & & & & & & \\
\hline Мати- $B * 052: 01$ & 54 & 63 & 1 & & & & & & & & & & & & & \\
\hline Мати- $B^{*} 055: 01$ & 180 & 65 & 1 & & & & & & & & & & & & & \\
\hline Мати- $B * 058: 02$ & 177 & 43 & 1 & & & & & & & & & & & & & \\
\hline Мати- $B * 064: 01$ & 179 & 56 & 1 & 44 & 31 & 1 & & & & & & & & & & \\
\hline Мати- $B * 073 g 1$ & & & & 129 & 19 & 1 & 140 & 60 & 1 & & & & 149 & 32 & 1 & $B^{*} 073: 01, B^{*} 073: 02$ \\
\hline Мати- $B^{*} 074 g 1$ & \begin{tabular}{|l|}
- \\
\end{tabular} & 82 & 1 & & & & & & & - & 18 & 1 & 16 & 48 & 1 & $B * 074: 01, B * 074: 02, B * 074: 03$ \\
\hline Mamu-B Minor Alleles & & & & & & & & & & & & & & & & \\
\hline Мати- $B^{*} 035 \_B^{*} 049 g 1$ & 161 & 92 & 1 & & & & & & & 293 & 37 & 1 & 261 & 80 & 1 & $B^{*} 035: 01, B^{*} 035: 02, B^{*} 049: 01$ \\
\hline Мати- $B * 053 \mathrm{~g} 1$ & & & & & & & & & & & & & 114 & 59 & 1 & $B * 053: 02, B * 053: 03$ \\
\hline Мати- $B^{*} 054: 01$ & 194 & 95 & 1 & & & & & & & & & & & & & \\
\hline Мати- $B * 054: 04$ & & & & & & & & & & 321 & 26 & 1 & & & & \\
\hline Мати- $B * 054: 05$ & & & & & & & & & & & & & 169 & 59 & 1 & \\
\hline Мати- $B * 057: 01$ & & & & & & & & & & & & & 104 & 41 & 1 & \\
\hline Мати- $B * 057: 06$ & & & & 59 & 24 & 1 & 113 & 61 & 1 & & & & 51 & 40 & 1 & \\
\hline Мати- $B^{*} 057 g 1$ & & & & & & & & & & 152 & 10 & 1 & & & & $B * 057$ alleles \\
\hline Мати- $B^{*} 063: 03$ & 198 & 74 & 1 & 122 & 29 & 1 & 184 & 73 & 1 & 243 & 16 & 1 & & & & \\
\hline Мати- $B * 063: 05$ & & & & & & & & & & & & & & 16 & & \\
\hline Мати- $B^{*} 063 g 1$ & 121 & 74 & 1 & & & & & & & & & & & & & $B^{*} 063: 01 \_$alleles, $B * 063: 02: 02 \_$allele \\
\hline Мати- $B * 070 g 2$ & 28 & 100 & 1 & & & & & & & & & & 89 & 68 & 1 & $B^{*} 070: 01, B * 070: 06$ \\
\hline Мати- $B * 072 g I$ & & & & 218 & 41 & 1 & 447 & 161 & 1 & 365 & 25 & 1 & 330 & 74 & 1 & $B^{*} 072 \_8 \_$alleles \\
\hline Мати- $B * 092 g I$ & & & & - & 35 & 1 & 14 & 88 & 1 & 23 & 25 & 1 & 82 & 84 & 1 & $B^{*} 092: 01, B * 092: 02, B * 092: 03$ \\
\hline Мати- $B * 098 g 1$ & 198 & 108 & 1 & & & & & & & 324 & 32 & 1 & 238 & 60 & 1 & $B * 098 \_9$ alleles \\
\hline Мати- $B * 109: 06$ & 479 & 92 & 1 & 479 & 49 & 1 & & & & & & & & 19 & & \\
\hline Мати- $B * 134 g 2$ & 257 & 82 & 1 & 276 & 24 & 1 & & & & & & & & & & $B^{*} 134: 03, B^{*} 134: 04, B^{*} 134: 06$ \\
\hline Мати- $B^{*} 188 g 1$ & & & & & & & 336 & 42 & 1 & 535 & 11 & 1 & & & & $B^{*} 188: 01, B * 188: 02$ \\
\hline Мати-B11L*01:12 & & & & - & 29 & - & 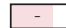 & 89 & 1 & & & & - & 74 & 1 & \\
\hline Мати-B11L*01g1 & - & 58 & 1 & - & 36 & 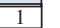 & - & 79 & 1 & - & 49 & 1 & & & & B11L*01_alleles,B11L*01:04,B11L*01:05 \\
\hline Мати-В16*01:01 & & & & 44 & 34 & 1 & 72 & 61 & 1 & & & & 110 & 29 & 1 & \\
\hline Мати-B16*01g1 & 181 & 74 & 1 & & & & & & & 176 & 23 & 1 & & & & B16*01_alleles \\
\hline Мати-B16*01g2 & & & & & & & 51 & 47 & 1 & \begin{tabular}{|l|}
77 \\
\end{tabular} & 14 & - & & & & $B 16 * 01: 02, B 16 * 01: 09$ \\
\hline Мати-В17*01g1 & & & & & & & & & & 1221 & 12 & 1 & 1227 & 17 & 1 & B17*01_14_alleles \\
\hline Мати-B17*01g2 & 1305 & 74 & 1 & 773 & 18 & 1 & 593 & 24 & 1 & 971 & 9 & 1 & & & & B17*01_9_alleles \\
\hline Мати-B17*01g4 & & & & & & & & 6 & & & & & & & & $B 17 * 01: 09, B 17 * 01: 15$ \\
\hline Мати-В20*01:02 & & & & & & & 304 & 43 & 1 & & & & & & & \\
\hline Мати-B20*01g1 & 176 & 66 & 1 & 181 & 21 & 1 & 272 & 82 & 1 & 365 & 33 & 1 & & & & $B 20 * 01: 01, B 20 * 01: 04$ \\
\hline Mamu-I Alleles & & & & & & & & & & & & & & & & \\
\hline Мати-I*01gI & 130 & 63 & 1 & 129 & 31 & 1 & 485 & 151 & 1 & 532 & 44 & 1 & 252 & 68 & 1 & I_01_66_alleles \\
\hline Мати-I*01g3 & & & & & & & & & & 81 & 28 & 1 & & & & $I^{*} 01: 19,1 * 01: 20: 01$ \\
\hline
\end{tabular}

Fig. 1 Comparison of MHC class I results from MiSeq PCR amplicon versus whole exome genotyping strategies for a representative breeding group of rhesus macaques 
bioRxiv preprint doi: https://doi.org/10.1101/625244; this version posted May 2, 2019. The copyright holder for this preprint (which was not certified by peer review) is the author/funder. All rights reserved. No reuse allowed without permission.

\begin{tabular}{|c|c|c|c|c|c|c|c|c|c|c|c|c|c|c|c|c|}
\hline $\begin{array}{l}\text { Relationship } \\
\text { Animal_ID }\end{array}$ & \multicolumn{3}{|c|}{$\begin{array}{c}\text { Dam } \\
\text { r05029 }\end{array}$} & \multicolumn{3}{|c|}{$\begin{array}{l}\text { Progeny } 1 \\
\text { r17099 }\end{array}$} & \multicolumn{3}{|c|}{$\begin{array}{l}\text { Sire } \\
\text { r07010 }\end{array}$} & \multicolumn{3}{|c|}{$\begin{array}{l}\text { Progeny } 2 \\
\text { r17041 }\end{array}$} & \multicolumn{3}{|c|}{$\begin{array}{l}\text { Progeny } 3 \\
\text { r17061 }\end{array}$} & \multirow{3}{*}{ Ambiguous Allele Group } \\
\hline Assay & MiSeq & IDSR S & AVAGE & MiSeq & DSR S & IVAGE & MiSec & DSR S & IVAGE & Miseq & DSR S & VAGE & MiSeq & DSR S & AVAGE & \\
\hline \multicolumn{16}{|l|}{ Mamu-DRB Alleles } & \\
\hline Mamu-DRB1 $1 * 03: 09$ & 198 & 370 & 1 & 72 & 131 & 1 & 170 & 305 & 1 & 272 & 107 & 1 & 156 & 200 & 1 & \multirow{3}{*}{ DRB 1*03:06,DRB $1 * 03: 26$} \\
\hline Mamu-DRB 1*03g1 & & & & 2789 & 217 & 1 & 2714 & 497 & 1 & & & & 3052 & 499 & 1 & \\
\hline Mamu-DRB $1 * 10: 03$ & & & & 1761 & 168 & 1 & 1952 & 358 & 1 & & & & 2711 & 310 & 1 & \\
\hline Mamu-DRB*W2g1 & 154 & 451 & 1 & 72 & 183 & 1 & 132 & 410 & 1 & 206 & 176 & 1 & 107 & 398 & 1 & \multirow{3}{*}{$\begin{array}{l}\text { DRB*W2:01,DRB*W2:05 } \\
\text { DRB*W3:03:01,DRB*W3:03:02 }\end{array}$} \\
\hline Mamu-DRB*W3g1 & 1936 & 546 & 1 & & & & & & & 3097 & 217 & 1 & & & & \\
\hline Mamu-DRB*W4:01 & 1777 & 414 & 1 & & & & & & & 2631 & 158 & 1 & & & & \\
\hline Mamu-DQA/DQB Alleles & & & & & & & & & & & & & & & & \multirow{5}{*}{ DQA $1 * 01: 04: 01, \mathrm{DQA} 1 * 01: 04: 02$} \\
\hline Mamu-DQA1*01:02 & & & & 2384 & 98 & 1 & 2444 & 256 & 1 & & & & 2964 & 154 & 1 & \\
\hline Mamu-DQA1*01g1 & 2298 & 318 & 1 & 2046 & 96 & 1 & 2276 & 288 & 1 & 4317 & 107 & 1 & 3360 & 210 & 1 & \\
\hline Mamu-DQA1*23:01 & 2883 & 334 & 1 & & & & & & & 5079 & 108 & 1 & & & & \\
\hline Mamu-DQB1*06:01 & 3803 & 160 & 1 & 3980 & 41 & 1 & 3892 & 152 & 1 & 5757 & 33 & 1 & 3892 & 122 & 1 & \\
\hline Mamu-DQB1*06g2 & & & & 5229 & 173 & 1 & 4784 & 345 & 1 & & & & 6400 & 282 & 1 & DQB $1 * 06: 05, \mathrm{DQB} 1 * 06: 18$ \\
\hline $\begin{array}{l}\text { Mamu-DQB1*18g4 } \\
\text { Mamu-DPA/DPB Alleles }\end{array}$ & 2656 & 329 & 1 & & & & & & & 4504 & 108 & 1 & & & & DQB $1 * 18: 02, \mathrm{DQB} 1 * 18: 27$ \\
\hline Mamu-DPA1*02g1 & 5450 & 1765 & 1 & 5301 & 316 & 1 & 5153 & 840 & 1 & 8727 & 758 & 1 & & & & DPA1*02_9_alleles \\
\hline Mamu-DPA1*04g1 & & & & 435 & 181 & 1 & 489 & 385 & 1 & & & & 1075 & 350 & 1 & DPA $1 * 04: 03: 01$,DPA $1 * 04: 03: 02$, DPA $1 * 04: 05$ \\
\hline Mamu-DPA1*11:01 & & & & & & & & & & & & & & 225 & 1 & \\
\hline Mamu-DPB1*02g1 & 106 & 25 & 1 & 87 & 73 & 1 & & & & & & & 342 & 47 & 1 & DPB $1 * 02: 01$, DPB $1 * 02: 04: 01$, DPB $1 * 02: 04: 02$ \\
\hline Mamu-DPB1*15g1 & 254 & 217 & 1 & 286 & 31 & 1 & 207 & 129 & 1 & 3084 & 60 & 1 & 1769 & 9 & & DPB $1 * 15: 01$, DPB $1 * 15: 02$ \\
\hline
\end{tabular}

Fig. 2 Comparison of MHC class II results from MiSeq PCR amplicon versus whole exome genotyping strategies for a representative breeding group of rhesus macaques 
A Relationship

GS ID
MHC Haplotype

MHC Haplotype 2

Mamu-A Major A
Mamu-A $I^{*} 00192$

Мати-AI**002:01

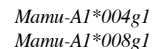

Мати-AI*008gI
Мати-AI*023 $A 1 * 106 \mathrm{~g} I$

Mamu-AI*023_Al*106g
Mamu-A Minor Alleles

Mamu-A2*05g1

Mати-A3*13_A4*I

Мати-A $3 * 13 g 1$

Mamu-A4*I 491
Mamu-E Alleles

Mamu-E Allels
Mamu-E* $02 g 1$

Мати-- $E^{*} 02 g 1$
Мати- $E^{*} 22 g 4$

Мати- $E^{*} 02 g 5$

Mamu- $E^{*} 02 g 7$
Mamu- $E^{*} 02 g 8$

Mamu-B Major Alleles

Мати- $B * 0019$

Мати- $B^{*} 012: 01$

Мати-B__022gI

Мати- $B^{*} 027 g 1$

Мати- $B * 030 \mathrm{gl}$

Мати- $B * 030 \mathrm{~g} 2$

Мати- $B^{*} 031: 03$

Мати- $B^{*} 041$ gI

Мати- $B * 043: 01$

Мати- $B^{*} 048: 01$

Мати- $B * 052: 01$

Мати--B*055:01

Мати-B* ${ }^{*} 064: 01$

Мати- $B * 073 g 1$

Мати- $B * 074 g 1$

Mamu-B Minor Alleles

Мamu- $B * 035$ - $B * 049$

Мати- $B * 054: 01$

Мати- $B$ *054:04

Мати-В*054:05

Мати- $B * 057: 01$

Мати- $B * 057: 06$

Мати- ${ }^{*} 063: 03$

Мати- $B * 063 g$

Мати- $B^{*} 063 \mathrm{gI}$
Мати- $\boldsymbol{B}^{*} 070 \mathrm{~g} 2$

Мати- $B * 072 g 1$

Мати $-B * 092 g 1$

Мати- $B * 098 g I$

Мати- $B * 13492$

Мати-B* $188 g 1$

Mamu-B1IL*01:12

Мати-B1IL*01g

Мати-В16*01:01
Мати-B16*01g1

Мати-BI6*01g2

Мати-В17*01g1

Мати-B17*01g2

Мати-В20*01:02

Mamu-B20*01gI

Mamu-I 1 Allgl

Мати- ${ }^{*} 01$ Ig 1

$\begin{array}{cccc}\text { Dam } & \text { Progeny } 1 & \text { Sire } & \text { Progeny } 2 \text { Progeny } 3\end{array}$

\begin{tabular}{rccccc} 
r05029 & r17099 & r07010 & r17041 & r17061 Ambiguous Allele Groups \\
\hline & a & b & $c$ & $b$ &
\end{tabular}

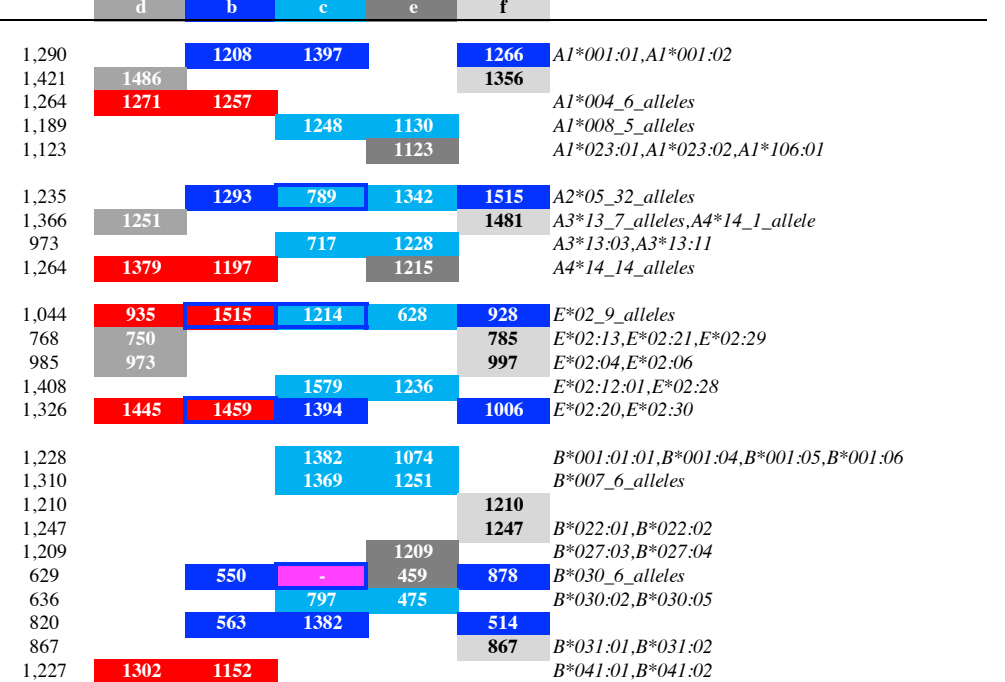

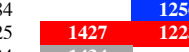

2223

$\begin{array}{lll}1276 & 1291 & 1311\end{array}$

1341

(1)

(1)

718
1,039
1,165

, 165

1,259
1,376
1,354
1,301

1,301

1,322
1,198
1,044
1,303

1,303

958
621

621
988
636
513

513
1,035

615
1,126

944
293
B

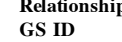

RS ID

MHC Haplotype 1

MHC Haplotype 2

Mamu-DRBI*03:09

Mamu-DRBI*03gI

Mamu-DRBI*10:03

Mamu-DRB*W2gl
Мати-DRB $D$ *W3g

Мати-DRB*W3g1
Мати-DRB*W4:01

Mamu-DQA/DQB Allele

Мати-DQA $1 * 01: 02$

Mamu-DQA $* 0191$

Мати-DQA* *23:01

Мати-DQB1*06:01

Мати-DQBI*-DQB1*1862

Mamu-DPA/DPB Alleles

Мати-DPAI*02gI

Мати-DPAI*04gI

Mamu-DPAT $11: 01$

Мати-DPBI*02gI

Мати-DPB1*16:01

\begin{tabular}{cccc} 
Dam & Progeny 1 Sire Progeny 2 Progeny 3 \\
\hline
\end{tabular}

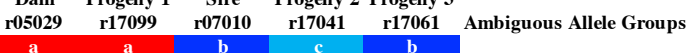

$\begin{array}{cccccc}\mathbf{a} & \mathbf{a} & \mathbf{b} & \mathrm{c} & \mathbf{b} \\ \mathrm{d} & \mathrm{b} & \mathrm{c} & \mathrm{c} & \mathbf{c}\end{array}$

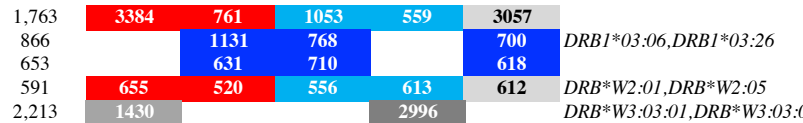

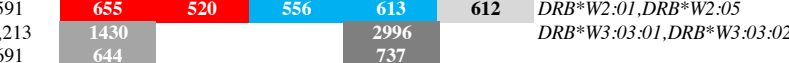

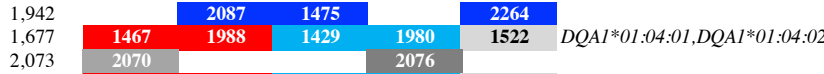

\begin{tabular}{|r|r|r|r|r}
1334 & 3199 & 4063 & 2780 & \\
\hline 1076 & 3257 & & 2975 & $D Q B 1 * 06: 05, D Q B 1 * 06: 18$
\end{tabular}

\begin{tabular}{l|l|l|l|l|l}
1,897 & 1989 & 1795 & 1929 & 1873 & $D P A *^{*} 02$ _a alleles
\end{tabular}

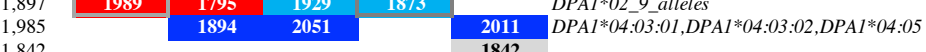

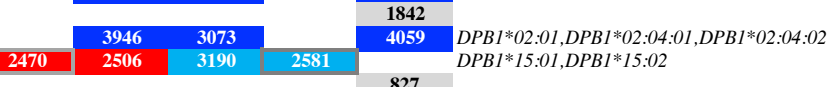

C MHC Haplotype

Mamu-DRB Haploty

Mamu-DQA

Mamu-DPA

Mamu-DPB

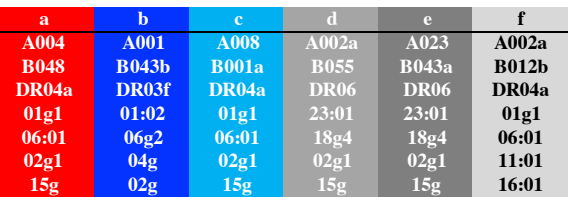

Fig. 3 Lengths of MHC sequence contigs assembled by the SAVAGE method for a representative breeding group of rhesus macaques. Values in the body of this figure indicate contig lengths generated for these MHC sequences in each animal.

(A) MHC class I genotyping results are illustrated for the five animals in this breeding group. Three false negative allele calls for SAVAGE versus the expected genotypes for these animals are highlighted in magenta, e.g., Mamu-B*030g1 in sire r07010. Sequences highlighted in red are associated with the maternal MHC Haplotype a that was inherited by Progeny 1 from its dam. Sequences in Progeny 1 and Progeny 3 for their MHC Haplotype b that was inherited from sire r07010 are highlighted in dark blue while the light blue sequences represent the alternate paternal Haplotype $\mathrm{c}$ that was inherited by Progeny 2. Three unique extended Haplotypes in this breeding group are indicated with shades of grey (d-f). MHC allele groups that are shared by both parental haplotypes are indicated by colored borders around filled cells.

(B) MHC class II genotyping results are illustrated for this same breeding group.

(C) Abbreviated Mamu haplotype designations are summarized for the sx extended

MHC haplotypes identified by segregation in this breeding group. For example, the twenty MHC sequences (red) that are associated with extended Haplotype a can be summarized by the following string of abbreviated Mamu haplotype designations: Mamu-A004/B048/DR04a/DQA01g1/DQB06:01/DPA02g1/DPB15g (Karl et al 2013). 
bioRxiv preprint doi: https://doi.org/10.1101/625244; this version posted May 2, 2019. The copyright holder for this preprint (which was not certified by peer review) is the author/funder. All rights reserved. No reuse allowed without permission.

Supplementary Table 1. Fraction of exome sequence reads corresponding to MHC class I and class II genes after target capture with the VCRome2.1 probe design alone

\begin{tabular}{|c|c|c|c|c|c|c|c|c|c|c|}
\hline GS ID & SRA Accession & $\begin{array}{c}\text { Total Raw } \\
\text { Reads }\end{array}$ & $\begin{array}{c}\text { MHC-I } \\
\text { Reads } \\
\text { Extracted }\end{array}$ & $\begin{array}{c}\text { DRB1/3/4/5 } \\
\text { Reads } \\
\text { Extracted }\end{array}$ & $\begin{array}{c}\text { DQA1 } \\
\text { Reads } \\
\text { Extracted }\end{array}$ & $\begin{array}{c}\text { DQB1 } \\
\text { Reads } \\
\text { Extracted }\end{array}$ & $\begin{array}{c}\text { DPA1 } \\
\text { Reads } \\
\text { Extracted }\end{array}$ & $\begin{array}{c}\text { DPB1 } \\
\text { Reads } \\
\text { Extracted }\end{array}$ & $\begin{array}{c}\text { MHC } \\
\text { Reads } \\
\text { Extracted }\end{array}$ & $\begin{array}{r}\text { MHC \% } \\
\text { of total }\end{array}$ \\
\hline cy0642 & SAMN11281734 & $79,795,404$ & 4,848 & 26,822 & 2,568 & 2,274 & 900 & 81,340 & 118,752 & 0.15 \\
\hline cy0643 & SAMN11281735 & $86,265,844$ & 4,408 & 26,930 & 2,542 & 2,320 & 936 & 74,370 & 111,506 & 0.13 \\
\hline cy0646 & SAMN11281736 & $109,909,444$ & 4,724 & 25,476 & 2,382 & 2,196 & 910 & 72,206 & 107,894 & 0.1 \\
\hline cy0648 & SAMN11281737 & $106,315,304$ & 5,558 & 34,442 & 2,966 & 2,714 & 1,092 & 97,254 & 144,026 & 0.14 \\
\hline cy0649 & SAMN11281738 & $78,575,038$ & 6,022 & 33,742 & 3,176 & 2,970 & 1,134 & 90,556 & 137,600 & 0.18 \\
\hline cy0651 & SAMN11281739 & $102,890,862$ & 6,686 & 33,194 & 3,388 & 3,094 & 1,294 & 94,532 & 142,188 & 0.14 \\
\hline cy0652 & SAMN11281740 & $89,074,544$ & 4,652 & 25,314 & 2,660 & 2,478 & 976 & 69,976 & 106,056 & 0.12 \\
\hline cy0654 & SAMN11281741 & $99,169,798$ & 5,294 & 30,328 & 2,924 & 2,756 & 1,042 & 79,664 & 122,008 & 0.12 \\
\hline & Average & $93,999,530$ & 5,274 & 29,531 & 2,826 & 2,600 & 1,036 & 82,487 & 123,754 & 0.13 \\
\hline
\end{tabular}

